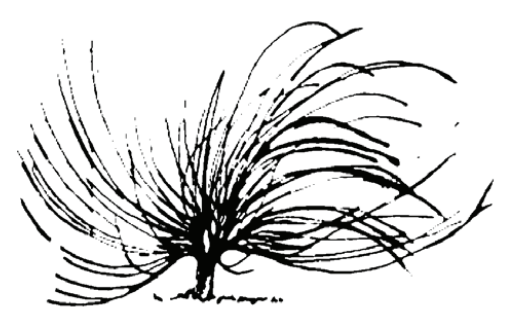

\title{
Malestar/Bienestar Docente: EI Caso de Tres Escuelas Interculturales Bilingües de Arantepacua y Capacuaro Michoacán, México
}

Giselle León León ${ }^{1}$

Universidad Nacional

Heredia, Costa Rica

giselle.leon.leon@una.cr

Patricia Serna González ${ }^{3}$

Universidad Pedagógica Nacional

Michoacán, México

patysernagonzalez@gmail.com

\author{
María de Lourdes Vargas Garduño ${ }^{2}$ \\ Universidad Michoacana de \\ San Nicolás de Hidalgo \\ Michoacán, México \\ luluvargas61@gmail.com
}

\begin{abstract}
Resumen
Con la intención de conocer la relación entre el malestar y bienestar docente como binomio opuesto y la asociación a factores personales, institucionales y sociales desde una perspectiva indígena-intercultural, se realizó un estudio en tres centros educativos de primarias interculturales bilingües: dos de Arantepacua y una de Capacuaro, ambos pueblos p'urhépechas del Estado de Michoacán. El estudio fue cualitativo fenomenológico, con realización de entrevistas a profundidad y una encuesta. Dentro de los resultados del
\end{abstract}

\section{(c) (i) $(5)$}

Recibido: 20 de enero de 2015 - Aprobado: 18 de agosto de 2015

1 Doctora en Educación. académica de la División de Educología, Universidad Nacional de Costa Rica.

2 Doctora en Ciencias Sociales. Profesora Investigadora de la Facultad de Psicología Universidad Michoacana de San Nicolás de Hidalgo, Michoacán, México.

3 Doctora en Ciencias de la Educación. Profesora Investigadora de la Universidad Pedagógica Nacional, asignatura escuela de psicología UMSNH, Michoacán, México 
estudio se resaltan la prevalencia de malestar principalmente en factores sociales tales como: la migración, disciplina, cambios en las políticas educativas y formación docente. Otro de los factores que evidenció malestar fue el institucional en relación con la sobrecarga de labores de los y las docentes. Por su parte se identificó bienestar en factores individuales ya que son autoeficaces.

Palabras clave: malestar, bienestar docente, educación indígena, interculturalidad

\begin{abstract}
With the purpose of knowing the relationship between the teachers' well-being and discontentment as an opposing pairing and the association to personal, institutional and social factors from an indigenous-intercultural perspective, a study was conducted in three bilingual intercultural primary education centers: two from Arantepacua and one from Capacuaro; both are P'urhépechas peoples from the Michoacán State. This was a qualitative phenomenological study using in-depth interviewing research and one survey. Among the results of this study, the prevalence of discontentment mainly in social factors, such as migration, discipline, changes in education policies and teacher training stand out. Another factor that demonstrates discontentment was the institutional one in relation to the teachers' excessive workload. On the other hand, well-being was identified in individual factors because they are self-sufficient.
\end{abstract}

Keywords: discomfort, teacher welfare, indigenous education, multiculturalism

\title{
INTRODUCCIÓN
}

Las comunidades indígenas actuales, si bien algunas conservan la herencia cultural de sus ancestros prehispánicos, no permanecen estáticas, sino que la modernidad y la posmodernidad han incidido en su dinamismo, lo que les ha implicado generar procesos de transformación social, para ajustarse a los requerimientos de las diversas épocas históricas que les ha tocado vivir. En México, desde la época colonial y sobre todo, a partir de la postrevolución 1925-1930), las políticas frente a los 
indígenas han sido contrarias; en lugar de respetar y promover su cultura, se les ha tratado de "castellanizar" y "mestizar", para que pudieran entrar en el "progreso". El resultado ha sido la desaparición de muchas lenguas, la pérdida de culturas y la tendencia a adoptar costumbres citadinas, olvidando lo propio, que es considerado como poco valioso.

En este contexto y en concordancia con varios movimientos latinoamericanos y europeos, surge en México, a principios del siglo XXI, la implementación de una política educativa tendiente a reivindicar los valores indígenas: la educación intercultural bilingüe, que caracteriza hasta la fecha a las escuelas de educación preescolar y primaria; algunas secundarias, pocos bachilleratos y unas cuantas universidades interculturales.

Todo lo anterior, unido a la globalización, ha afectado el bienestar social y emocional de las personas. El ritmo de vida acelerado, las obligaciones, las exigencias, las presiones sociales y profesionales, las tensiones y el aumento de responsabilidades, se han reflejado en la vida cotidiana de los y las docentes indígenas. En relación con esta situación, resulta relevante hacer notar que existe un aumento alarmante de enfermedades psicosociales asociadas al trabajo y derivadas de distintos fenómenos, como el estrés, el mobbing, el acoso, la violencia; esas consecuencias del estrés se han convertido en nuevos desafíos para algunos profesionales, particularmente, en aquellos que se desempeñan en el ámbito de la salud, del trabajo social y la enseñanza (personas que trabajan con personas). Esta situación ha sido muy estudiada en contextos citadinos, pero poco en ámbitos indígenas.

A pesar de responder a los cambios que se dan en la sociedad, los grupos indígenas de manera general y en particular los docentes, promueven una serie de tradiciones que las políticas públicas con frecuencia han modernizado, pero no en un sentido dialógico, intercultural, elementos como los mencionados y el mismo contexto sociocultural en que se desarrolla el proceso educativo pueden generar malestar en los docentes. Ante este panorama, resulta indispensable el desarrollo de estudios que den a conocer el entorno sociocultural y pedagógico en que se está desarrollando la docencia intercultural bilingüe en dos comunidades P'urhepechas pertenecientes a la zona de la Meseta o de la Sierra.

Existen estudios previos, como el de García (2004), que abordan el desarrollo histórico de lo que ha sido la educación indígena, tomando como referencia el caso de Michoacán. Destaca la etapa de la castellanización y las políticas asimilacionistas, hasta llegar a la implantación de la 
educación intercultural bilingüe (EIB). En esa misma temática, se puede hacer alusión a la investigación titulada La Educación Intercultural Bilingüe y la vivencia de la interculturalidad en familias p'urhepechas. El caso de Arantepacua, Municipio de Nahuatzen, Michoacán (Vargas-Garduño, 2013), en el cual se hace una evaluación del impacto de la EIB a partir de un estudio etnográfico con familias y de etnografía de aula. En relación con estudios sobre malestar o bienestar docente, si bien existen varios, no se identificó ninguno que tomara en cuenta las comunidades indígenas. Tal como hemos dicho, nuestro interés tiene dos componentes que se conjugan para comprender de qué manera la implementación de la EIB se vincula con una vivencia de malestar docente y qué factores favorecen el bienestar docente en el contexto p'urhepecha.

Por esta razón, este artículo describe una investigación realizada de manera interdisciplinaria, en el marco de una estancia realizada en la Facultad de Psicología de la Universidad Michoacana San Nicolás de Hidalgo por parte de la autora costarricense, asesorada por las dos autoras mexicanas, que desarrollan su investigación en las comunidades de Arantepacua y Capacuaro, Michoacán. El eje de la experiencia relatada se centra en la siguiente interrogante: ¿Qué factores individuales, sociales e institucionales se pueden identificar en los discursos expresados por los docentes p'urhepechas participantes acerca de su vivencia de malestar/ bienestar docente, en escuelas primarias que están dentro del modelo de la educación intercultural bilingüe? A partir de esa interrogante, surge como objetivo de estudio: Identificar algunos factores: personales, institucionales y sociales asociados a la percepción de bienestar/malestar docente en docentes de tres centros educativos P'urhepechas.

Para lograr este objetivo, en un primer momento se hace una reseña de las comunidades indígenas en estudio, luego se describe el eje teórico principal: el malestar/bienestar docente, en un tercer momento se indica cómo se realizó la investigación y por último se presenta un análisis descriptivo de la realidad investigada.

\section{EL CONTEXTO: EL PUEBLO P'URHEPECHA}

En México hay 105556 hablantes de p'urhepecha, de los cuales el 92\% residen en el estado de Michoacán (Instituto Nacional de Geografía y Estadística, INEGI, 2009). La región p'urhepecha es la más extensa de las zonas indígenas que existen en el Estado de Michoacán 
y se localiza en la parte central de este. Aunque existe un debate acerca de cómo deben ser nombrados los pobladores indígenas mayoritarios de Michoacán, los diversos investigadores han encontrado principalmente dos términos: tarascos y p'urhepecha. La decisión sobre cuál es el correcto, aún es motivo de debate (Márquez, 2007). Con respecto del término tarascos, la versión más difundida es la que relata que cuando llegaron los conquistadores españoles en el año 1522, los miembros de la corte procuraron ganarse su amistad, dándoles a sus hijas como mujeres y los llamaron tarascue (tarasïkue), que en su lengua quiere decir yerno (Alcalá, 1540/2008). Por este motivo, los españoles empezaron a llamarles tarascos, con lo cual se difundió este nombre en todos los ámbitos y durante muchos años.

Por otra parte, el término p'urhepecha ha sido rescatado por algunas personas profesionales $p$ 'urhepechas, a quienes a partir del año 1930 se les ha dado la oportunidad de participar en ámbitos institucionales y académicos. En opinión de estos académicos p'urhepechas, su gentilicio significa gente o personas, es lo que los define correctamente porque resulta una autoafirmación, como seres humanos. No obstante, otros académicos refieren que el nombre procede de $p$ 'urejkuni que quiere decir poner a prueba a alguien para realizar una tarea; de ahí se derivó p'urhep, en singular y p'urhepecha, en plural que significa gente que está probada para realizar una tarea. Por tanto, desde su punto de vista, la designación no corresponde a gente o persona, en general, sino a gente trabajadora (Vargas-Garduño, 2013).

\section{Reseña de Arantepacua}

El nombre de la comunidad p'urhepecha de Arantepacua posee dos significados aceptados por sus pobladores, la primera es Jarhanpakua que deriva de las raíces p'urhépecha jarhan (estar o haber) у tepacua (plan, por lo que significa "estar en el plan", referido a que la comunidad está en un lugar plano). La segunda, Arantepacua, corresponde al nombre castellano, la cual fue tomada de Aranti, en honor al nombre del rey fundador, uniendo esta raíz con la palabra tepacua, el significado podría ser el plan del Rey Aranti o el lugar plano del Rey Aranti; esta última es la más aceptada en dicho pueblo (Vargas-Garduño, 2013).

Con respecto de la religión, la gran mayoría de las personas de la comunidad profesan el catolicismo. Las fiestas principales de la 
comunidad giran en torno a las celebraciones religiosas y de la escuela. Arantepacua se divide en dos barrios conocidos como barrio primero y el segundo. Cuenta con luz eléctrica en las casas y alumbrado público, el abastecimiento del agua se da por pozos, distribuido en tuberías. No se cuenta con servicio de alcantarillado.

En la comunidad hay una plaza o jardín principal en donde se ubica una especie de quiosco, llamado pérgola, ${ }^{4}$ en la cual se llevan a cabo las actividades propias de la comunidad: fiestas civiles y religiosas y diversos eventos durante el año. El pueblo cuenta con una iglesia, dedicada a la Virgen de la Natividad y un auditorio que tiene una cancha de basquetbol. En cuanto a servicios educativos, existe educación inicial, un preescolar, dos primarias, una telesecundaria y una extensión del Colegio de Bachilleres cuya sede es Cherán.

Hay una caseta telefónica, una tienda que recibe el correo ${ }^{5}$ y varios equipos de sonido distribuidos en varios puntos estratégicos de la comunidad, los cuales cumplen la importante función social de informar sobre eventos, ventas especiales, felicitaciones, entre otras cosas. En cuanto al transporte, hay una ruta de autobuses y taxis que van a Uruapan y a Pátzcuaro o a Nahuatzen, saliendo de la plaza de la comunidad. Asimismo, hay transportes colectivos que van hacia Nahuatzen.

El mayor porcentaje de las casas está construido de cemento y tabique. Casi todas las viviendas cuentan con servicios de agua y luz, y con aparatos eléctricos como radio, televisión, licuadora y algunos microondas; algunas de las casas poseen teléfono.

La mayoría de los pobladores son indígenas p'urhepecha, salvo algunas personas que han venido de otras comunidades, principalmente, por haberse casado con algún varón de la comunidad. La lengua materna es el p'urhepecha, que es utilizada por la mayor parte de la población. Aunque la gente habla muy bien el p'urhepecha, muy pocos son los que saben leerlo y menos aún, escribirlo. Asimismo, las mujeres adultas suelen usar su vestuario tradicional, aunque esta costumbre se está perdiendo a causa de los elevados costos y de la influencia de la escolarización y la migración. Es notoria la tendencia de los pobladores a dejar su cultura para "mestizarse" y "progresar".

4 En prácticamente todas las comunidades y municipios de la Meseta P'hurépecha existe una pérgola. 5 Ahí llega el correo para los habitantes del pueblo, quienes pagan por recogerlo. 


\section{Reseña de Capacuaro}

El nombre de la comunidad "Capacuaro" posee dos significados: el primero desde la religión, en la cual se dice que proviene de la capa de cuero de San Juan Bautista, el segundo y más aceptado por los pobladores, es que proviene de la palabra K'apakun la cual significa, rodear o abrazar, ya que esta comunidad está rodeada de montañas, por lo que su traducción al español sería "Lugar resguardado".

Con respecto de la religión, al igual que en Arantepacua, la gran mayoría de las personas de la comunidad profesan el catolicismo. Las fiestas principales de la comunidad giran en torno a las celebraciones religiosas y de las escuelas. La comunidad cuenta con luz eléctrica en las casas y alumbrado público, el abastecimiento del agua se da por pozos, distribuido en tuberías. No hay servicio de alcantarillado.

El mayor porcentaje de las casas está construido de cemento y tabique. Casi todas las viviendas cuentan con servicios de agua y luz y con aparatos eléctricos. En la comunidad hay una plaza o jardín principal, en la cual se llevan a cabo las actividades propias de la comunidad: fiestas civiles y religiosas y diversos eventos durante el año. La iglesia del pueblo está dedicada a San Juan Bautista, de ahí que el 24 de junio sea la fiesta patronal.

Capacuaro cuenta con preescolar, tres primarias (dos de ellas de doble turno) y una secundaria, una clínica del Instituto Mexicano de Seguro Social (IMSS) y clínicas particulares de medicina, seguridad pública y tiendas así cómo vías de acceso nacionales e internacionales. A nivel del transporte, se cuenta con taxis que dan servicio de Uruapan y Paracho.

Los pobladores en su mayoría son p'urhépecha hablantes de su lengua materna. Una de las problemáticas sociales es la inmigración de algunos pobladores por falta de espacios de trabajo. Un rasgo característico del pueblo es que la gran mayoría de las mujeres usan su vestuario tradicional, que es el mismo que en Arantepacua. A pesar de ser un pueblo con casi el doble de pobladores que en Arantepacua, se encuentra entre dos ciudades importantes, Capacuaro es un pueblo que ama y defiende su cultura. 


\section{EL MALESTAR DOCENTE}

La expresión malestar docente se comenzó a usar con mayor frecuencia en los años 80 , "se emplea para describir los efectos permanentes de carácter negativo, que afectan las personalidad del enseñante como resultado de los cambios acelerados producidos en el contexto histórico-social" (Fueguel y Montoliu, 2005, p. 37). Tomando como referencia la cita anterior, se puede indicar que el Burnout o síndrome de "quemado por trabajo" o "de desgaste profesional" puede generarse si no se atiende oportunamente el malestar docente (Bermejo y Prieto, 2005).

Por su parte, Esteve (2011) define malestar docente como el resultado de las condiciones psicológicas y sociales en que se ejerce la docencia en la actualidad, las cuales desencadenan actitudes y sentimientos negativos que afectan el desempeño y la percepción del rol profesional y del centro educativo donde se trabaja. Si bien no existen estadísticas fiables sobre esta patología, se calcula que en sectores como la enseñanza, un $30 \%$ de las bajas se deben a este malestar, al igual que un elevado porcentaje de las que se dan entre los profesionales de la salud (Cornejo y Quiñonez, 2007).

Esteve (2011) uno de los autores que ha investigado esta problemática, incluye como promotores del malestar docente, las variables propias del sujeto tales como la existencia de motivaciones negativas que le llevaron a ser docente y las características psicológicas del sujeto, lo que le lleva a afirmar que muchos individuos llegan ya al ejercicio profesional de la docencia proclives al malestar, por lo que a continuación se indican una clasificación de los factores negativos que pueden influir en profesional de la docencia, a partir de autores como Tonon (2003); Travers y Coopers (1997); León (2011) y Napione (2008), quienes los clasifican en factores sociales, institucionales e individuales. A continuación se describirá cada uno de ellos de manera teórica, para en un apartado posterior, analizar cómo se apreciaron estos factores en los profesores participantes.

\section{Factores del malestar docente}

Algunos autores que han escrito sobre el malestar docente, específicamente sobre los factores, proponen diferentes clasificaciones, por ejemplo, Moriana y Herruzo (2004) lo han clasificado en tres grupos: 
variables sociodemográficas (edad, sexo, estado civil, nivel impartido, tipo de centro, entre otras), variables de personalidad (actitudes y tipo de personalidad) y variables propias del trabajo y organizacionales (sobrecarga laboral, trabajo administrativo, número de alumnos, entre otros.). A partir de esa clasificación y otras como las propuestas por León (2011), Feixas (2004), Cornejo y Quiñones (2007) y Esteve (2011), las autoras del artículo proponen los siguientes factores:

\section{Factores sociales}

Al docente en la actualidad se le han asignado una serie de responsabilidades o roles dados por la demanda social, tales como atender a los conflictos grupales y necesidades individuales de los educandos, los cuales responden -como ya se mencionó- a la globalización y demandas sociales, las cuales no han sido acompañadas por una adecuada formación psicopedagógica, medios y recursos didácticos, cambios administrativos, entre otros. El docente es considerado como el culpable y responsable de las situaciones negativas del sistema educativo, recibe poco apoyo de los padres, quienes en la mayoría de los casos salen en defensa de sus hijos, independientemente de la situación.

Asimismo, la imagen social del docente cada vez es más cuestionada, ya que existe una influencia por los estereotipos de los medios de comunicación que presentan una imagen idealizada de la enseñanza o una imagen controversial, a partir de la cual se producen una serie de opiniones en torno a qué es, cómo se hace y qué se debe hacer (Esteve, 2011).

Otro componente social que también se puede considerar pedagógico es la formación idealista, que se da en las universidades que forman al futuro profesional de la docencia. Esto consiste en procesos educativos centrados en proyecciones ideales en las cuales los educandos están anuentes al proceso de aprendizaje, sin enseñarle qué estrategias y recursos debe emplear cuando la situación no es la ideal. En este caso, el educador principiante se puede encontrar con que la realidad es otra y que ésta no corresponde a los esquemas ideales con los que se le han formado. Esa situación puede llevar a que el docente quiera dejar la docencia (Esteve, 2011). A partir de esto, se hace necesaria una formación integral docente que abarque las áreas de la especialidad, pedagógica y psicosocial, de una manera holística y apegada a la realidad, para que se desempeñe de la mejor manera. 


\section{Factores institucionales}

Los factores institucionales se asocian al hecho de que el docente realiza su trabajo en organizaciones que responden a un esquema de burocracia profesionalizada. Como señala Feixas (2004), la cultura institucional influye de manera importante para generar bienestar o malestar entre los docentes. Desde las instituciones, se generan dificultades a causa de la manera en que se ejerce el poder y la jerarquización. Para evitar ser promotoras de malestar docente, estas organizaciones requerirían de una distribución democrática del poder, de forma que el propio profesional ejerza el control sobre su trabajo y las decisiones que le afectan.

Entre los elementos que constituyen la cultura institucional resulta fundamental contemplar la relación con sus superiores, como otro de los factores que pueden generar malestar o bienestar entre los docentes. Dichas relaciones, por lo general son de carácter burocrático, muy ligadas al control/sanción y poco vinculadas al trabajo docente; lo cual va unido al excesivo papeleo y a una escasa participación en la resolución de sus problemas laborales o profesionales, así como las conflictivas relaciones con padres y compañeros.

Además de la multiplicidad de funciones cuyo cumplimiento se exige año con año al docente, mucho del trabajo del docente lo consume la cantidad de documentos que debe presentar, planeamientos, planillas administrativas, informes del rendimiento escolar, informes de los estudiantes con necesidades educativas especiales.

El edificio, las instalaciones y el mobiliario conforman el ámbito dentro del cual se establece las relaciones en las que se desarrolla la tarea educativa. Como espacio material, el edificio y sus instalaciones conforman un conjunto de condiciones que pueden afectar la calidad de la enseñanza y del aprendizaje. Por una parte, generan sensaciones de comodidad o incomodidad, seguridad o peligro, potencialidad o carencia y con ellas, comprometen la posibilidad o dificultad de los sujetos para sentirse, en mayor o menor grado, "predispuestos" frente a las demandas del trabajo.

Un componente más se asocia con lo que Cornejo y Quiñones (2007) 1lama "gestión del tiempo", puesto que con frecuencia los docentes se viven con sobrecarga laboral, no sólo en términos de cantidad de trabajo que deben realizar las personas profesionales de la educación 
cada día, sino también de la cantidad de tareas que deben llevarse a sus casas, interfiriendo en sus vidas privadas, sin mencionar todas las tareas inherentes al cargo, reuniones, efemérides, documentación de los educandos, actividades para recolectar fondos, organizar festivales, clausuras, desfiles, kermeses, exposiciones, concursos, asambleas de consejo técnico, presentación de exámenes, cursos de actualización (que con frecuencia pagan por su cuenta), realizar reuniones de evaluación con padres de familia, atender necesidades, quejas, peticiones de padres de familia, atender a directivos, administrativos, supervisión, jefatura de sector, entre otros.

\section{Factores individuales}

Las características de la personalidad configuran un elemento que debe ser considerado en relación con el malestar/bienestar. Por ejemplo, las personas entusiastas, con mayor dedicación profesional, susceptibles a identificarse con los problemas de los demás, sensibles, obsesivos y con baja autoestima pero a la vez, excesivamente idealistas, pueden ser proclives al malestar docente. Estas características son propias del patrón de personalidad tipo A (Manassero, et al. 2003).

Por su parte, el patrón B se caracteriza por ser relajado, tranquilo, abierto a las emociones, incluidas las hostiles. En este patrón predomina la tranquilidad y el equilibrio ante las diferentes situaciones. En cambio, el patrón de conducta tipo $\mathrm{C}$ se presenta en sujetos introvertidos, obsesivos, que interiorizan su respuesta al estrés, pasivos, resignados y apacibles, extremadamente cooperadores, sumisos y conformistas, siempre controlando las expresiones, deseosos de aprobación social (León, 2011).

Otro factor individual son las expectativas personales que se tengan en relación con el trabajo, algunas de las más frecuentes, en el ámbito de la enseñanza, son las siguientes: la expectativa de que todos los estudiantes tendrán una respuesta positiva ante la intervención personal. Además, el profesor espera que su trabajo sea apreciado y valorado por sus compañeros y superiores inmediatos, especialmente por los estudiantes y sus padres, entre otros (León, 2011).

De igual manera, se puede indicar el manejo del estrés como un factor, ya que si bien es parte de la vida, se debe hacer un equilibrio entre el estrés y el "distrés". A partir de lo indicado, afirmamos que los factores individuales se pueden asociar con la autoeficacia 
del docente, es decir, la relación entre pensamiento, acción y emoción (Bandura, 2011).

\section{BIENESTAR DOCENTE}

Se define bienestar emocional como "el grado como una persona juzga favorablemente la calidad global de su vida" (Bisquerra, 2008, p. 228). Por tanto, el bienestar al igual que el malestar puede ser influenciado por factores como los antes descritos, por consiguiente, el bienestar docente se puede decir que es el cúmulo de satisfacciones que genera el proceso educativo, las cuales se traducen en satisfacción por trabajar con personas, agrado por lo que se hace, elemento que permite trasmitir ese gusto a los educandos, así como saber mediar con los componentes sociales que se presentan de manera que se tome de ellos lo que enriquece el proceso educativo.

Lo descrito se refleja en situaciones como: saber sacar provecho del apoyo social de toda la comunidad educativa, autorregulación, autoeficacia percibida (Fernández, 2008), manejo de tiempo escolar y de esparcimiento, estrategias de afrontamiento de situaciones estresantes entre otros.

\section{METODOLOGÍA}

La investigación se enmarcó en el enfoque cualitativo dentro del diseño fenomenológico, pues como lo indica Álvarez-Gayou (2013), en la presente investigación las investigadoras realizaron una interpretación del sentir de los docentes en relación con el proceso educativo de las comunidades educativas de Arantepacua y Capacuaro, ubicadas en la Meseta P'urhepecha.

Se empleó el método fenomenológico considerando que los docentes, actúan en función del significado que tienen del proceso educativo. Sus posturas son productos sociales, su significado se conoce a través de su decir, de su sentir, la actitud reflexiva, crítica del investigador, guía la obtención del dato sin adulteración, gracias a la epojé y a la aplicación de la reducción eidética, que permite llegar a esencias generales a partir de hechos particulares (Lambert, 2006). Esta metodología permite entrar al mundo de la vida de alguien (Morse, 2006); en este caso de los docentes, en su experiencia cotidiana para lograr un rescate 
de su conciencia hacia el malestar y bienestar del proceso educativo, en el marco del proceso educativo intercultural bilingue.

Para lograr el objetivo de la investigación, se implementó una encuesta y una entrevista en profundidad. La encuesta constó de 10 preguntas y la entrevista se realizó con base en seis preguntas-guía ambas versaron sobre los factores (sociales, institucionales y personales) que inciden en la generación de bienestar o de malestar en los docentes en el marco del proceso educativo aprendizaje intercultural bilingüe. Los instrumentos y técnicas fueron aplicados a los docentes en los centros educativos en sus tiempos libres y en el marco de una capacitación que estaban recibiendo los docentes por parte de la Dra. Vargas, coautora de este texto.

Con respecto de las consideraciones éticas, se invitó a los docentes a participar voluntariamente en la experiencia que aquí se relata, a partir del consentimiento informado. En el caso particular de la entrevista, se solicitó a los participantes su autorización para grabar la conversación. Para resguardar la confidencialidad de los datos, a lo largo del texto, se usan las siguientes abreviaturas: DM, docente masculino; DF, docente femenino; E, entrevistadora.

Para realizar este estudio, se contó con el aporte de 23 docentes, de los cuales 15 fueron hombres y ocho mujeres de tres escuelas primarias interculturales bilingües: Mariano Matamoros de la comunidad de Capacuaro; Vasco de Quiroga y Aranti de la comunidad de Arantepacua, quienes en el momento del estudio se desempeñaban como docentes frente a grupo.

\section{HALLAZGOS DEL ESTUDIO}

Dado que, el eje de la investigación se centra en los factores que inciden en la generación de bienestar o de malestar en los docentes en el proceso de enseñanza aprendizaje intercultural bilingüe, conceptos que se han explicado antes, en este apartado se desglosarán las manifestaciones de dichos factores que los profesores p'urhepechas manifestaron en las entrevistas y encuestas. Por tanto, las categorías se construyeron de acuerdo con la misma lógica antes presentada: factores sociales, institucionales y personales. 


\section{Factores sociales, asociados a situaciones que se viven en su entorno y les provocan sensaciones de malestar o bienestar}

En relación con esta categoría malestar/bienestar a nivel del contexto social se identificó que algunos docentes encuestados expresaron malestar en situaciones como los cambios en las políticas educativas en relación con modelos tomados de otras realidades educativas. En ese sentido, se expresaron ideas tales como que la educación indígena tiene particularidades que no son tomadas en cuenta y no se respetan, que los currículos son dictados desde un escritorio, ya que por ejemplo en el caso del modelo por competencias se tomó de países desarrollados y no se adecua ni se brinda una adecuada capacitación a los docentes. Asimismo, se quejan de que el material bibliográfico (libros de texto), llega a estas comunidades después de mucho tiempo, como lo expresó el docente A en la entrevista.

$D M-A$ : en cuanto a los planes y programas, pues como ya ve los modifican, por ejemplo, cuando yo estudié teníamos otro programa matriz integración humana y ahora es el famoso mentado desarrollo por competencias yo no le veo nada..."

E- entonces usted considera que se propone en el papel únicamente DM-A: exactamente pues a lo mejor es la misma apatía

E- apatía a qué

DM-A: a la misma comodidad de no seguir estudiando, que no te deja ir más allá...

Tal como se evidencia en el discurso del maestro, existe malestar en relación con la forma en que se plantean las políticas educativas, ya que si bien a nivel teórico las políticas educativas indican las capacidades de los gobernantes y de la sociedad para construir posibilidades de un futuro mejor para sus habitantes, no se está tomando en cuenta las características de las poblaciones indígenas ni mucho menos siendo congruentes con el modelo de educación intercultural bilingüe que se supone están aplicando en su contexto.

Tomando en cuenta que la educación es la vía indiscutible para lograr mejores condiciones de vida para todas las personas que habitan en el planeta, mediante el desarrollo de mecanismos sociales de inclusión. En ese sentido, se demuestran ideas contradictorias con el afán 
de lograr una educación de calidad, a partir de modelos externos a la cultura indígena. No obstante, el afán modernizador es el que afecta las decisiones de los jefes de Estado, lo que se traduce en tendencias sociales asimilacionistas con respecto de los grupos indígenas; se habla de propiciar el desarrollo, eliminar el rezago, entre otros, idea, que a pesar de lo indicado, se rige con ideales educativos que no responden a sus necesidades (Vargas-Garduño, 2013).

En relación con el apoyo por parte de los padres de familia en una de las comunidades particularmente en una de las escuelas, los maestros y las maestras refirieron que no existe apoyo por parte de los padres de familia: "Los padres no asumen su papel, la educación al $100 \%$ le corresponde al maestro. Dicen los padres, por eso lo mando a la escuela para que se eduque, yo no puedo decirle nada al hijo, péguele... (DM- M)"

A partir de lo expresado por ese docente, se evidencia la existencia de malestar asociada a factores sociales, ya que el contexto sociocultural de desintegración familiar y pérdida de valores se refleja en la escuela; así como la creencia de los padres de familia indígenas de que es tarea de la escuela la educación de sus hijos. Como lo resalta León (2011), muchas de las responsabilidades relacionadas con las actitudes y el comportamiento de los estudiantes han recaído sobre los docentes, sin que la familia y la sociedad asuman paralelamente su compromiso.

Por otro lado, en una de las comunidades se identificó malestar social producto de la migración de los padres de familia, a causa de la falta de oportunidades de empleo locales, por lo cual se deben trasladar hacia otros lugares en algunos casos llevándose a sus niños, dejando interrumpido el proceso educativo, o en su efecto, depositando en la madre la responsabilidad de velar por sus hijos(as), en relación con este particular, el participante DM-S indicó:

DM- S: Hay días en que sólo tengo cuatro estudiantes, por la migración y porque a las madres no les hacen caso,

E: ¿Cómo es que no les hacen caso?

$D M$ - S: como el padre se va a otros lugares a vender las cosas de madera, se quedan con sus madres, ella los mandan a clases, pero no les hacen caso y salen de la casa pero se quedan en la plaza.... 
Siguiendo con factores sociales, uno de los que presentó mayor recurrencia fue la falta de formación docente para adecuarse a la realidad educativa del contexto. En ese sentido, la mayoría de los docentes entrevistados indicaron:

DF-B: Bueno desde niña siempre quise ser yo maestra. La idea cambia cuando a uno se le presentan problemas, uno piensa que todo es fácil, no va a hacer ningún problema, pues pero surgen diferentes problemáticas con los niños, niños que no aprenden, niños rebeldes, pues para eso no nos forman, pues en lo único que cambia, quiera uno que nos dieran todas esas herramientas pero no es posible porque esos problemas se presentan en el trabajo diario.

DM-C: Ha cambiado mucho pues porque la idea inicial, era dar clases y atender a los alumnos y hasta ahí, no es sólo pararse en el aula, sino saber enseñar, saber organizarse, aparte cómo enseñar, qué estrategias usar, cómo responder a los diferentes alumnos son diferentes en cada comunidad.

DF- LL: Es un cambio muy grande, pues como estudiante de la normal tenía muchas ideas, voy a hacer esto voy a cambiar eso, y tenía hasta formas de cómo trabajar, cuando ya llego al trabajo con los niños es un poquito más complicado, porque existe una gran diversidad aunque sea un grupo hay niños que avanzar más rápido, hay niños que tardan mucho y se atrasan más, peor si es numeroso y la idea es muy diferente en lo teórico.

Tal como se muestra, la formación universitaria representa un componente que puede favorecer la tendencia hacia el malestar, ya que cuando las y los docentes inician su formación en las aulas universitarias pueden recibir suficiente preparación teórica para desempeñarse en su profesión; no obstante, los programas de formación del profesorado carecen de cursos enfocados al análisis y al aprendizaje de estrategias para lidiar con situaciones y condiciones laborales difíciles, tales como las socioafectivas, relacionadas con el manejo del estrés, comunes a la mayoría de los y las docentes. Esta desvinculación entre la preparación profesional y la realidad de la práctica hace que el profesorado, cuando se enfrenta a este tipo de situaciones, se sienta desorientado y muchas veces imposibilitado para actuar, por lo que le resulta difícil encontrar soluciones a los problemas y a las situaciones de su labor diaria. 
Otro de los factores que evidenció malestar fue la educación intercultural bilingüe (EIB), tanto para los profesionales que manejan la lengua materna como para los que no lo hacen, puesto que, como lo describe Vargas-Garduño (2013), en la formación inicial casi no se les brindan herramientas para comprender los fundamentos teóricos de la EIB, ni mucho menos cómo aplicarlos en el aula. A lo más que llega la conciencia de ello es a usar la lengua p'urhepecha en la enseñanza. En relación con esto expresaron:

DM- G: Yo soy de la comunidad y cuando voy clases lo hago en p'urhepecha pero algunos padres no quieren, porque dicen que no quieren que sus hijos sean discriminados....

DF-S: Algunos alumnos dicen que no hable así, yo les digo que es una materia más....

A pesar de lo expuesto antes, algunos con posturas positivas y otros con negativas todo(as) encuestados(as) externaron la importancia de rescatar su cultura; sin embargo, algunas personas docentes no manejan la lengua materna, elemento que a algunos(as) padres les agrada para que castellanicen a sus hijos. En contraste, algunos docentes sí se preocupan por el rescate de valores y tradiciones locales, contextualizando en sus clases a partir de la realidad en la que viven y haciéndoles ver a sus educandos el valor como seres humanos que tienen, en ese sentido un docente indicó:

$D M-C$ : Usamos una palabra en este contexto que es lengua p'urhepecha "kachumbekua", que significa "no son cultos", pues han perdido esa cultura de valores, de que son irrespetuosos, yo creo que hay un problema en la transición de la comunidad, porque han llegado muchos profesionistas y de acuerdo a eso de pasar de una cultura a otra que se van los profesionistas, como que traen consigo esto la perdida de muchos valores, a veces el aislamiento es mejor, porque si una comunidad está aislada en esa comunidad no entran a contaminar.

E: Eso, ¿Usted lo ve totalmente negativo?

DM-C: Totalmente negativo, es negativo porque aunque se vuelva profesional debe rescatar todo lo bueno de la comunidad. 


\section{Factores personales, entendidos como el conjunto de características de cada individuo que le permiten salir adelante (autoeficacia)}

Pese a las manifestaciones anteriores de malestar, se identificaron factores de bienestar del docente a nivel individual que se manifiestan en el proceso educativo institucional como es la autoeficacia, reflejada en la vocación del maestro. Tal como lo refieren Bermejo y Prieto en el año 2005, los profesores con elevado sentimiento de autoeficacia manifiestan gran entusiasmo y vocación por el proceso educativo de sus alumnos, razón por la cual presentan mayor apertura a los cambios, tienen la disponibilidad de aplicar métodos y técnicas diferentes siempre que estas permitan mejorar el proceso de mediación pedagógica, planifican y organizan mejor sus clases y dedican tiempo a los estudiantes a pesar de tener necesidades educativas, quieren aprender. Siguiendo con el contraste entre las contradicciones del sistema educativo y la vocación del docente se muestra la postura de una maestra que indicó:

DF-D: Bueno los padres de familia, bueno digo, por los comentarios que hacen, están a gusto con mi trabajo, me da gusto que cuando reparten los grupos ellos dicen que quieren que estén conmigo..., en cuanto los compañeros he recibido felicitaciones en cuanto a mi trabajo. Pues me gusta hacer lo que hago, pues y es el reflejo de los comentarios de mis compañeros, padres de familia y de los niños y que si lo alienta a uno a seguirse preparando a seguir estudiando a todo.

\section{Factores institucionales, manifestados en la percepción del clima organizacional de la institución educativa}

El párrafo anterior resalta como el ser humano es un ser integral, es decir, pese a que existen presiones sociales tales como los cambios en las políticas educativas, el hecho de que se tenga una buena actitud hacia el proceso educativo evidencia bienestar en el docente. Al respecto Assman (2002) propone que el proceso de aprendizaje ocurre en un ambiente ameno, plácido y motivador, lo cual se apreció en la escuela de Capacuaro, aunque no en las otras dos.

Esta motivación favorece el desarrollo de una conciencia de aprendizaje vivencial, la cual se logra gracias a la comunicación, la 
relación humana y la aventura de realizarse como persona, a partir de la creatividad, la investigación y el intercambio de experiencias, competencias que se reflejan en la docente entrevistada, al referir gustarle lo que hace, por ende, cada día tratar de hacerlo de la mejor manera, tanto por responder a las necesidades de la comunidad educativa, como de sus propias convicciones de seguir aprendiendo para enriquecer su mediación pedagógica.

Vale resaltar que todos(as) los maestros y maestras de la investigación indicaron tener agrado por su profesión y por rescatar parte de su propia cultura. Pese a eso, no todos(as) hablan su lengua nativa, pero dicen apoyarse en los educandos que sí son bilingües, para poder profundizar en particularidades de la clase que por haberlas planteado en español no son comprendidas por algunos de los educandos.

Reforzando la postura anterior, se comparte lo que indicó una docente de una de las comunidades en relación con la autoeficacia y el reflejo de ella en el clima institucional: "Me gusta trabajar en este turno, porque el director da más libertad en el trabajo con los niños y eso me compromete en darle resultados..." (DF-F).

Tal como se evidencia, el tener un superior inmediato con el que se pueda hablar, que esté anuente a que sus docentes se superen, genera un clima organizacional más agradable y un mayor compromiso en la mediación pedagógica desarrollada en el aula, ya que si el docente no está motivado, debe hacer un esfuerzo doble al dar sus clases elemento que a la postre puede ser percibido por sus educandos.

Contrastando la postura anterior, algunos de los docentes de la otra comunidad tomada en cuenta en la investigación expresaron inconformidad por la poca presencia de su superior, al referir: “...el director debe ser más autoritario, debe asumir su trabajo,... debe estar más presente, para que pueda tener una mejor idea de cómo es nuestro trabajo (DM- L)".

Como se indicó antes, el proceso educativo se debe desarrollar a partir del aporte de los diferentes actores; en el caso de los líderes se requieren personas que consigan traducir el liderazgo en acciones que permitan desarrollar un clima organizacional agradable que se traduzca en procesos educativos de calidad. A partir de la postura antes descrita, se requieren líderes con presencia, ya que si bien se podría pensar que hay agrado por trabajar en total libertad, aducen requerir la guía, acompañamiento y orientación para que el clima institucional sea el idóneo. 
Siguiendo con algunos de los factores institucionales evidenciados en el estudio, a pesar de ser comunidades rurales, la mayoría de los y las docentes indicaron disponer de medios y recursos didácticos para enriquecer el proceso de enseñanza y aprendizaje; en contraste mostraron malestar hacia la cantidad de trabajo extracurricular con el que se debe cumplir. Al respecto León (2011), indica que la profesión docente es una de las carreras en las cuales existen muchas actividades inherentes al cargo, así como tareas extra horario lectivo. En ese sentido, al respecto indicó un docente "las cuestiones administrativas nos quitan tiempo de las clases, los registros de inscripción de la Secretaría de Educación que hay que estar completando... (DM- G)".

\section{REFLEXIONES FINALES}

Retomando la pregunta y el objetivo de la investigación, concluimos que se cumplieron, puesto que se lograron identificar algunos factores: personales, institucionales y sociales asociados a la percepción de bienestar/ malestar docente en docentes de tres centros educativos P'urhepechas.

Dentro de los factores que presentaron mayor malestar está el social, manifestado en elementos tales como: la disciplina de los educandos, la migración de padres de familia que implica ausentismo escolar en sus hijos y la formación idealista y descontextualizada que se le da en las universidades y escuelas formadoras de docentes. Otro de los factores sociales identificados fueron los cambios en las políticas educativas, específicamente, la implementación de un currículum que procede de un modelo de educación intercultural bilingüe para el que no se les prepara. En consecuencia, los docentes aplican el estilo asimilacionista a la cultura hegemónica, que fue el que aprendieron en su vivencia como estudiantes. Así, aunque se cuente con un modelo educativo aparentemente adecuado al contexto, no se sigue por falta de preparación para ello.

Por su parte, los factores individuales reflejaron bienestar, ya que los docentes son autoeficaces, les agrada ser docentes y rescatar su cultura. Uno de los elementos que reflejó un tanto de malestar fue la dificultad para manejar la lengua materna; algunos, en tanto que no la dominan, y los demás, porque saben hablarla muy bien y entenderla, pero no pueden escribirla ni leerla; mucho menos enseñar a los niños a leer y escribir en p'urhepecha. 
Con respecto de los factores que evidenció malestar fue el institucional en relación con la sobrecarga de labores de los docentes y la falta de una cultura organizacional que favorezca la gestión del tiempo. En el mismo ámbito de los factores institucionales, algunos docentes refirieron bienestar por el tipo de administración, basada en el diálogo y la motivación, mientras que, por el contrario, algunos refirieron malestar por la ausencia de guía por parte de su superior inmediato.

Entre las limitaciones del estudio, se encontró sobre todo la limitación de tiempo, debido a la corta estancia de investigación de la autora principal de este artículo, lo que dificultó la profundización en la experiencia del personal docente de las tres escuelas participantes. No obstante, al tratarse de una temática poco investigada en el ámbito indígena, lo aquí referido permite repensar la posibilidad de generar una línea de investigación sólida en este terreno, dado que otras investigaciones no han considerado este contexto intercultural para trabajar este tema del malestar/bienestar. No podemos pensar que un docente solo vive malestar o solo bienestar, en uno mismo hay factores duales, es decir, en algunos se siente y se percibe malestar en otros, expresa bienestar; son elementos coexistentes y con perdurabilidad.

La educación debe afrontar sin más demora los desafíos de este siglo, y es en las políticas educativas donde debe quedar plasmado, cómo se van a enfrentar esos cambios. A partir de la reflexión hecha en el documento, se hace un llamado a la sociedad en general y a los gobernantes en particular a tomar en cuenta el malestar/bienestar en ellas, de manera que se responda a las necesidades de las personas profesionales de la educación y de la sociedad, para lograr mayor efectividad en la educación intercultural.

\section{Referencias bibliográficas}

Alcalá, J. (1540/2008). Relación de Michoacán. Disponible en: http:// bibliotecadigital.ilce.edu.mx/sites/fondo2000/vol1/relacion/ $\mathrm{html} /$ indice.html

Álvarez-Gayou, J.L. (2013). Cómo hacer investigación cualitativa. Fundamentos y metodología. México, DF: Paidós.

Assman, H. (2002). Placer y ternura en Educación. Hacia una sociedad aprendiente. Madrid, España: Nárcea Ediciones. 
Bandura, A. (2001). Guía para la construcción de escalas de autoeficacia. Recuperado de, http://www.uky.edu/ eushe2/Pajares/effguideSpanish.html

Bermejo, L. y Prieto, M. (2005). Creencias irracionales en profesores y su relación con el malestar docente. Recuperado de http://dialnet. unirioja.es/servlet/articulo?codigo $=1147371$

Bermejo, L. y Prieto, M. (Septiembre-diciembre, 2005). Malestar docente y creencias de autoeficacia del profesor. Revista Española de pedagogía. 232, 493-510. Recuperado de http://revistadepedagogia.org/2007060263/vol.-1xiii-2005/n\%C2\%BA-232-septiembre-diciembre-2005/malestar-docente-y-creencias-de-autoeficacia-del-profesor.html

Bisquerra, R. (2008). Educación para la ciudadanía y convivencia. Madrid: Wolters Kluwer.

Cornejo,R. y Quiñonez,M. (Diciembre, 2007).Factores asociados al malestar/bienestar docente. Una investigación actual. Revista Electrónica Iberoamericana sobre Calidad, Eficacia y Cambio en Educación. (5), 5e, 75-80.

Esteve, J. (2011). El malestar docente. (6 impresión). Barcelona, España: Papeles de Pedagogías, Paidós.

Feixas, M. (2004). La influencia de factores, personales, institucionales y contextuales en la trayectoria y el desarrollo docente de los profesores universitarios. Educar (33), 31-35.

Fernández, M. (octubre, 2008). Bournout, autoeficacia y estrés en maestros peruanos: tres estudios fácticos. Ponencia presentada en el $2^{\circ}$. Foro de las Américas en Investigación sobre factores psicosociales de estrés y salud mental en el trabajo. Lima, Perú.

Fueguel, C. y Montoliu, R. (2005). El malestar docente, propuestas creativas para reducir el estrés del profesorado. Barcelona, España: Octaedro.

García, S. (enero-marzo,2004.) De la educación indígena a la educación bilingüe intercultural. La comunidad p'urhépecha, Michoacán, México. Revista Mexicana de investigación educativa, 20 (9), 61-81. Recuperado de http://www.redalyc.org/articulo. oa? id $=14002005$

Instituto Nacional de Geografía y Estadística. (2009). Perfil sociodemográfico de la población que habla lengua indígena. Recuperado de http://www.inegi.org.mx/prod_serv/contenidos/espanol/ 
bvinegi/productos/censos/poblacion/poblacion_indigena/leng _ indi/PHLI.pdf

Lambert, C. (2006). Edmund Husserl: La idea de la fenomenología. Teología y vida, 517-529.

León, G. (enero- junio,2011). Los profesionales de secundaria, como factores de riesgo en el síndrome de Burnout. Revista electrónica Educare, 15 (1), 177-191.Recuperado de http://www.revistas. una.ac.cr/index.php/EDUCARE/article/download/890/816

Manassero, M., Vazquez, A., Ferrer, V., Fórnes, J. y Fernández, C. (2003). Estrés y Bournout en la enseñanza. Palma, Universidad de las Illes Balears.

Márquez, P. (2007). ¿Tarascos o p'urhepechas? Voces sobre antiguas y nuevas discusiones en torno al gentilicio michoacano. México: El Colegio de Michoacán.

Moriana, Juan y Herruzo, Javier. (2004). Estrés y burnout en profesores. International Journal of Clinical and HealthPsychology, 4(3), 597-621. Morse, J. (2006). Asuntos críticos en los métodos de investigación cualitativa. Antioquía: Universidad de Antioquia.

Napione, M.E. (2008). ¿Cuándo se quema el profesorado de secundaria? España: Díaz de Santos.

Tonon, G. (2003). Calidad de vida y desgaste profesional: una mirada del síndrome de Burnout. Buenos Aires: Espacio.

Travers, A. y Cooper, C. (1997). El estrés de los profesores. La presión en la actividad docente. Barcelona: Paidós.

Vargas-Garduño, M. L. (2013). La educación intercultural bilingüe y la vivencia de la interculturalidad en familias p'urhepecha. El caso de Arantepacua, Municipio de Nahuatzen, Michoacán, México: Secretaría de Educación Pública/Coordinación General de Educación Intercultural y Bilingüe. 